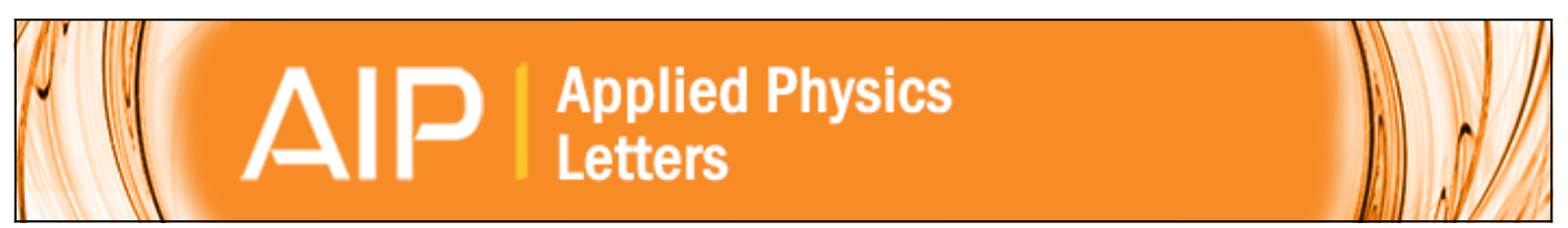

The roles of metallic rectangular-grating and planar anodes in the photocarrier generation and transport of organic solar cells

Wei E. I. Sha, Wallace C. H. Choy, and Weng Cho Chew

Citation: Applied Physics Letters 101, 223302 (2012); doi: 10.1063/1.4768236

View online: http://dx.doi.org/10.1063/1.4768236

View Table of Contents: http://scitation.aip.org/content/aip/journal/apl/101/22?ver=pdfcov

Published by the AIP Publishing

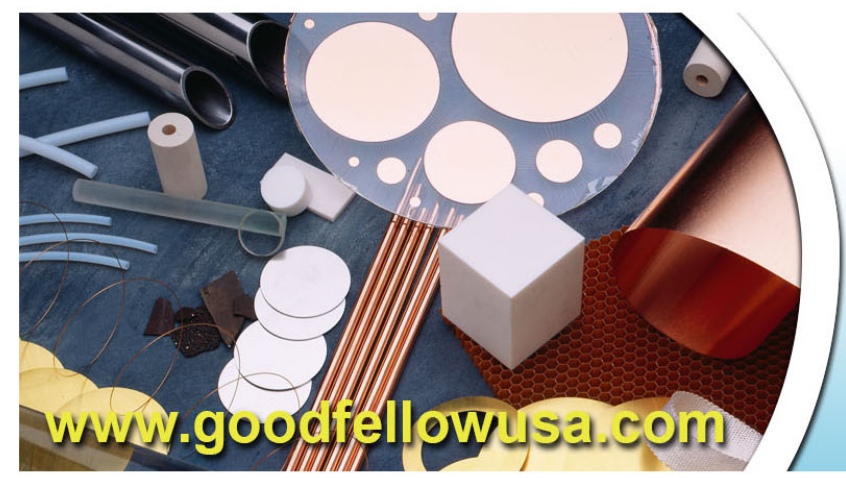

BODIFEl|OII

metals • ceramics $\cdot$ polymers composites $\bullet$ compounds $\bullet$ glasses

Save $5 \%$ B Buy online 70,000 products $\cdot$ Fast shipping 


\title{
The roles of metallic rectangular-grating and planar anodes in the photocarrier generation and transport of organic solar cells
}

\author{
Wei E. I. Sha, ${ }^{1}$ Wallace C. H. Choy, ${ }^{1, a)}$ and Weng Cho Chew ${ }^{2, b)}$ \\ ${ }^{1}$ Department of Electrical and Electronic Engineering, The University of Hong Kong, \\ Pokfulam Road, Hong Kong \\ ${ }^{2}$ Department of Electrical and Computer Engineering, University of Illinois, Urbana-Champaign, \\ Illinois 61801, USA
}

(Received 26 August 2012; accepted 4 November 2012; published online 27 November 2012)

\begin{abstract}
A multiphysics study carries out on organic solar cells (OSCs) by solving Maxwell's and semiconductor equations simultaneously. By introducing a metallic rectangular-grating as the anode, surface plasmons are excited resulting in nonuniform exciton generation. Meanwhile, the internal E-field of plasmonic OSCs is modified with the modulated anode boundary. The plasmonic OSC improves $13 \%$ of short-circuit current but reduces $7 \%$ of fill factor (FF) compared to the standard one with a planar anode. The uneven photocarrier generation and transport by the grating anode are physical origins of the dropped FF. This work provides fundamental multiphysics modeling and understanding for plasmonic OSCs. (C) 2012 American Institute of Physics.

[http://dx.doi.org/10.1063/1.4768236]
\end{abstract}

As one of the promising candidates for photovoltaic applications, organic solar cells (OSCs) have drawn considerable attention recently due to their properties of large-area production, mechanical flexibility, and low-cost processing. ${ }^{1,2}$ However, the short lifetime and diffusion length of excitons result in ultrathin active-layer configuration in OSCs. The configuration limits the light absorption efficiency and thereby the power conversion efficiency. The power conversion efficiency (PCE) of OSCs is given by $\mathrm{PCE}=J_{s c} V_{o c} \mathrm{FF} / P_{i n}$, where $J_{s c}$ is the short-circuit current, $V_{o c}$ is the open-circuit voltage, FF is the fill factor (FF), and $P_{i n}$ is the incident photon power. Having unique features of tunable resonance and unprecedented near-field concentration, plasmonics is one of enabling techniques for boosting the optical absorption of OSCs. ${ }^{3-6}$ The enhanced optical absorption substantially increases the generation rate of photocarriers and thus shortcircuit current. The basic device physics of OSCs has been investigated in literatures. ${ }^{7,8}$ However, the physical mechanism of the modified electrical properties of OSCs due to the introduction of metallic nanostructures has not been unveiled systematically. Apart from the optical absorption properties, ${ }^{3-6}$ the electrical properties of plasmonic OSCs, such as internal E-field distribution, recombination loss, and exciton dissociation, will greatly affect the performance of organic solar cell (OSC) devices.

In this paper, we model the optical and electrical properties of OSCs with the metallic grating and planar anodes through solving Maxwell's equations and semiconductor equations simultaneously. As a unique feature of OSCs, the exciton dissociation process can be reproduced by our multiphysics model that is different from the established models for silicon solar cells. ${ }^{9-11}$ The photocarrier's generation, transport, and collections are fully observed and comparatively studied for plasmonic OSCs with the gating anode and standard OSCs with the planar anode.

\footnotetext{
${ }^{a)}$ Electronic mail: chchoy@eee.hku.hk.

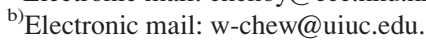

We will report peculiar electrical features of plasmonic OSCs and reveal the physical origin of the dropped FF. Regarding our previous work, ${ }^{12}$ we investigated the optical and electrical responses of OSCs with the metallic nanostrip anode. Critically different from the modulated boundary of the grating anode studied in this work, the flat boundary of the nanostrip anode induces uniform internal (electrostatic) E-field distribution in the active layer and thus unchanged FF. Furthermore, the modulated boundary of the grating requires more sophisticated numerical techniques to handle the boundary conditions encountered in semiconductor equations. The spatially uneven exciton generation and internal E-field distribution resulting, respectively, from the optical and electrical responses of the metallic grating should be taken into account and carefully manipulated for future designs of plasmonic OSCs. The multiphysics modeling and understanding are imperative for high-efficiency organic photovoltaic technique and low-cost green energy industry.

The schematic standard and plasmonic OSC structures are shown in Figs. 1(a) and 1(b), respectively. The blend active layer of bulk heterojunction OSCs comprises a small bandgap donor of PBDTTT-C-T (poly\{[4,8-bis-(2-ethylhexyl-thiophene-5-yl)-benzo[1,2-b:4,5-b']dithiophene-2,6diyl]-alt-[2-(2'-ethyl-hexanoyl)-thieno[3,4-b]thiophen-4,6diyl]\}) and an acceptor of $\mathrm{PC}_{70} \mathrm{BM}([6,6]$-phenyl C71butyric acid methyl ester). A silver rectangular-grating is introduced as the anode for the plasmonic OSC. Fig. 1(c) depicts the energy level diagram of the OSCs. The anode is assumed to be an ohmic contact while the cathode has an injection (Schottky) barrier of $0.2 \mathrm{eV}$. We use unified finitedifference method to model the multiphysics features of OSCs through solving Maxwell's equations and semiconductor equations (Poisson, continuity, and drift-diffusion equations) simultaneously. For the details about the algorithms and modeling parameters (See Secs. S1 and S2 of supplementary material ${ }^{13}$ ).

Figure 1(d) shows the ratio of the generation rate of the plasmonic OSC to that of the standard OSC. The extremely 
(a)
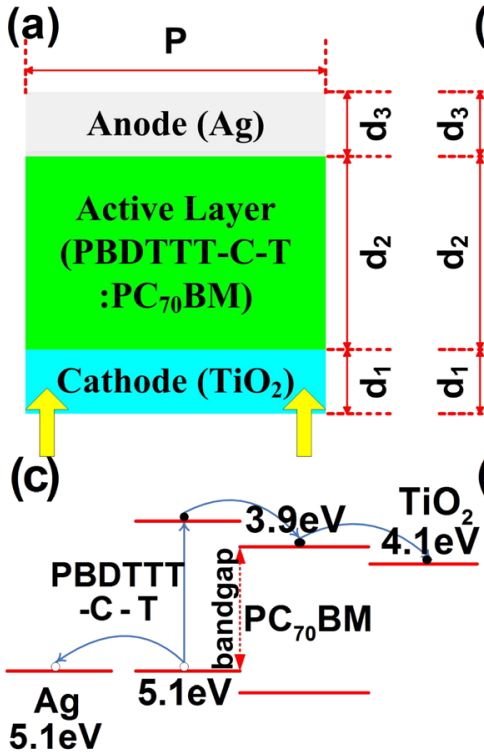

(b)

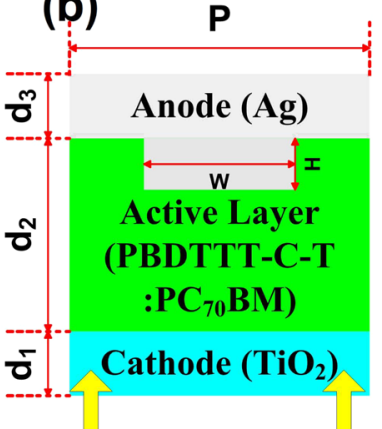

(d)

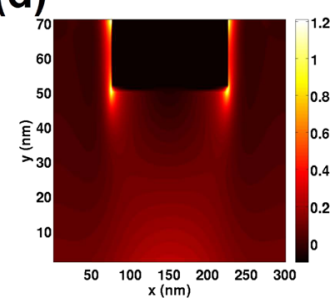

FIG. 1. (a),(b) The schematic patterns for the unit cells of the standard and plasmonic OSCs, respectively. The geometric parameters are $d_{1}=30 \mathrm{~nm}$, $d_{2}=70 \mathrm{~nm}, d_{3}=30 \mathrm{~nm}, P=300 \mathrm{~nm}, W=150 \mathrm{~nm}$, and $H=20 \mathrm{~nm}$. (c) The energy levels of active materials and electrodes. (d) The generation rate map of the plasmonic cell divided by that of the standard cell (in the active layer). The logarithmic scale is adopted.

dense exciton generation can be found around the metallic grating. Moreover, the increased exciton generation can be observed in the active region below the ridge of the grating. Floquet modes supported by the periodic grating will provide additional momentum to enable the excitation of surface plasmon by light which propagates along the interface between the silver grating and active material. From the reciprocity theorem in electromagnetics, the surface plasmon waves cannot be completely trapped in the grating layer and will damp in the active layer and escape into the air region. Hence, constructive interferences between surface plasmon waves and the incident light enhance the optical absorption and exciton generation in the active region below the ridge of the grating. An alternative explanation is that the grating scatters light back into the active layer where it may be trapped by the total internal reflection into other types of waveguide modes. From Fig. 1(d), the exciton generation is extremely nonuniform in the active layer of the plasmonic cell.

Figs. 2 and 3 show the potential distribution, recombination rate, electron and hole current densities at the shortcircuit condition for the standard and plasmonic OSCs, respectively. It can be seen from Fig. 3(a) that the internal E-field, which is the gradient of potential, presents uneven spatial distribution for the plasmonic cell remarkably different from the standard cell as illustrated in Fig. 2(a). The internal E-field at the center region between the ridge of the grating and opposite cathode section is stronger than the internal E-field at the two side regions between the troughs of anode and opposite cathode sections. The peculiar internal E-field distribution induces the nonuniform recombination rate at the cathode as shown in Fig. 3(b). It is because the free electrons experience different sweep-out time. A strong internal E-field results in short sweep-out time and low recombination. Regarding the drift current proportional to

(a)

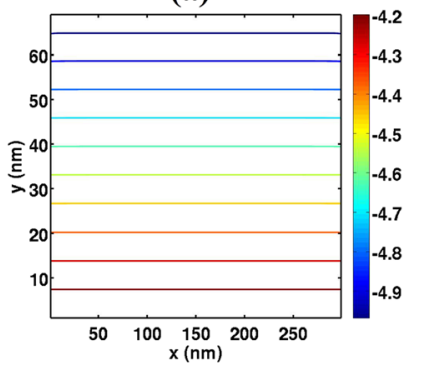

(c)

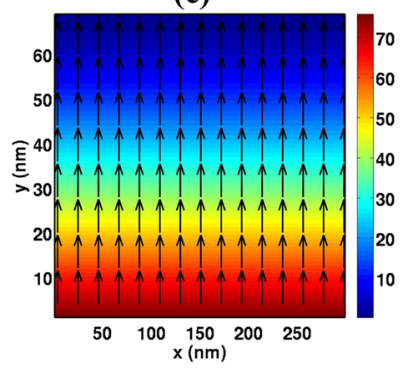

(b)

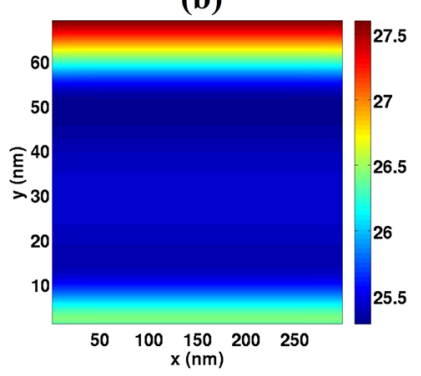

(d)

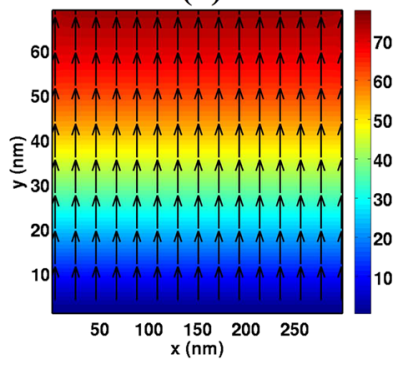

FIG. 2. The electrical results of the standard OSC at the short-circuit condition. (a) equipotential lines (V); (b) recombination rate with the logarithmic scale $\left(\mathrm{m}^{-3} \mathrm{~s}^{-1}\right)$; (c), (d) electron and hole current densities $\left(\mathrm{A} / \mathrm{m}^{2}\right)$. The color and arrow denote the amplitude and direction of the currents.

the internal E-field and charge concentration, the nonuniform electron current density with pronounced peaks at $x=50$ and $x=250 \mathrm{~nm}$ can be expected in the plasmonic cell as shown in Fig. 3(c). For the standard cell, Fig. 2(b) shows that the recombination near the anode is significantly larger than that near the cathode although the large generation rate is found near the cathode as depicted in Fig. S3 of Sec. S3 of supplementary material. ${ }^{13}$ In modeling, hole mobility has one order of magnitude lower than electron mobility, which is the case for most polymer materials. Holes have long sweep-out time and hopping-distance to migrate towards the anode. As a (a)

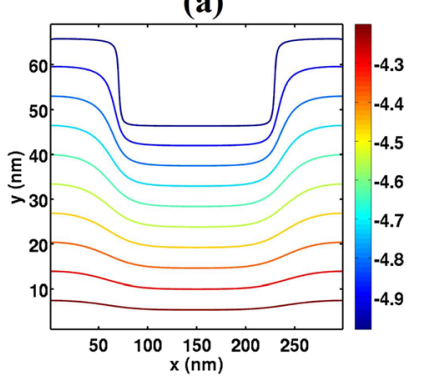

(c)

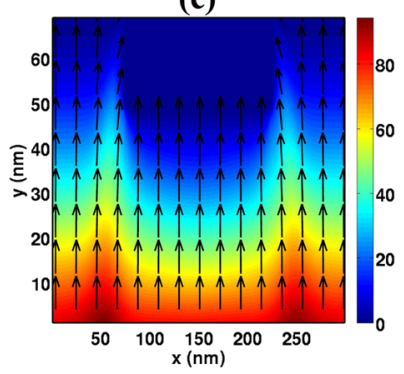

(b)

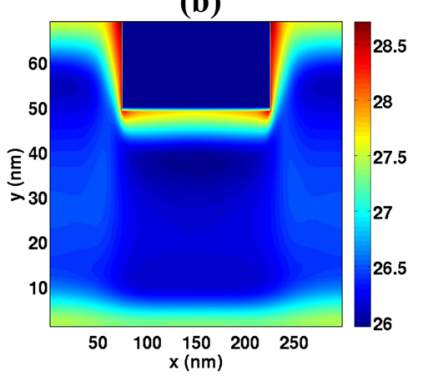

(d)

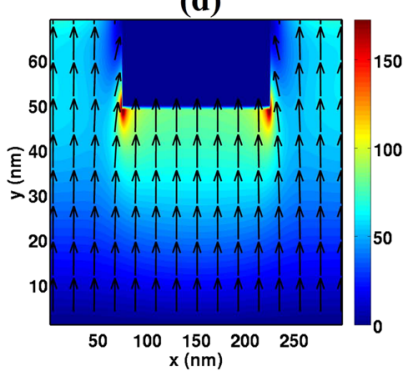

FIG. 3. The electrical results of the plasmonic OSC at the short-circuit condition. (a) Equipotential lines (V); (b) recombination rate with the logarithmic scale $\left(\mathrm{m}^{-3} \mathrm{~s}^{-1}\right)$; (c),(d) electron and hole current densities $\left(\mathrm{A} / \mathrm{m}^{2}\right)$. The color and arrow denote the amplitude and direction of the currents. 
result, the recombination loss of holes is larger than that of electrons. For the plasmonic cell, the recombination at the grating ridge and sides (especially at the junctions) drastically increases in contrast to the recombination at the grating troughs (see Fig. 3(b)). This feature agrees with the nonuniform exciton generation very well as illustrated in Fig. S4 of Sec. S3 of supplementary material. ${ }^{13}$ The huge recombination quenches a lot of free photocarriers and reduces the hole current density. Fig. 3(d) shows the hole current density of the plasmonic cell. We can see that the amplitude of the hole current density can be ignored near the junctions. Interestingly, the peak amplitude of the hole current density is noticeably larger than that of the electron current density owing to the favorable hole transport and thus lower recombination (see Figs. 3(c) and 3(d)). The holes generated around the grating can be collected by the anode due to the short hopping path and sweep-out time. Contrarily, the electron and hole current densities of the standard cell have almost the same peak amplitude as presented in Figs. 2(c) and 2(d).

Figs. 4 and 5 present the electrical responses of both cells at the open-circuit condition. The near zero internal E-field induces increased recombination loss and makes the charge extraction difficult. Meanwhile, the field-dependent exciton dissociation probability decreases leading to a large carrier loss as listed in Table I. Distinguishing from the injection barrier associated with the cathode, the ohmic anode has more free holes that cannot recombine with their electron counterparts. Therefore, the higher concentration of equipotential lines is observed near the anode for both cells (Figs. 4(a) and 5(a)). For the standard cell, the hole recombination regions occupy almost the whole active layer as shown in Fig. 4(b). For the plasmonic cell, besides the recombination peaks at the lateral sides and ridge corners of the grating, there is also a strong recombination at the active layer region below the grating as shown in Fig. 5(b). The (a)

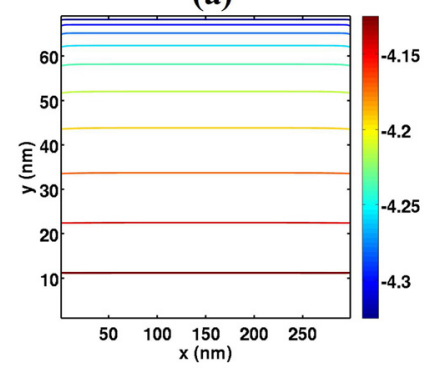

(c)

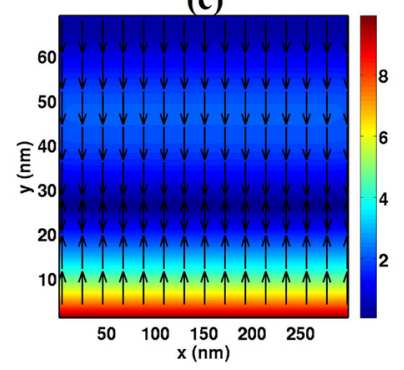

(b)

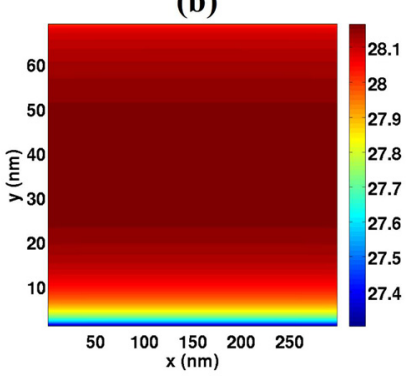

(d)

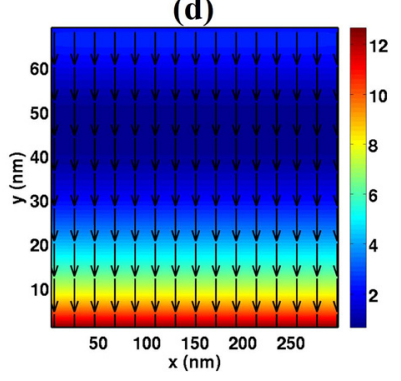

FIG. 4. The electrical results of the standard OSC at the open-circuit condition. (a) Equipotential lines (V); (b) recombination rate with the logarithmic scale $\left(\mathrm{m}^{-3} \mathrm{~s}^{-1}\right)$; (c),(d) electron and hole current densities $\left(\mathrm{A} / \mathrm{m}^{2}\right)$. The color and arrow denote the amplitude and direction of the currents. (a)

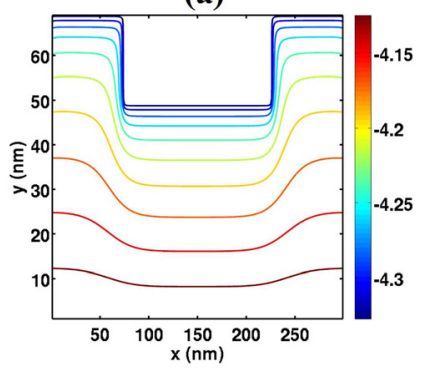

(c)

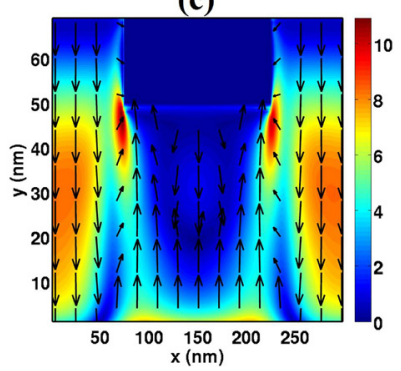

(b)

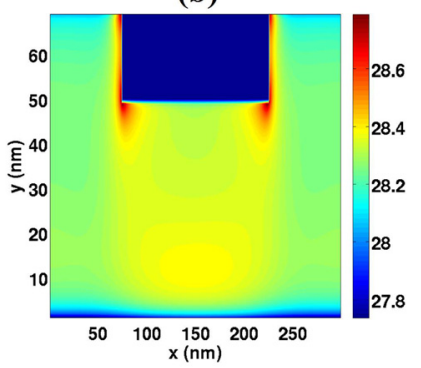

(d)

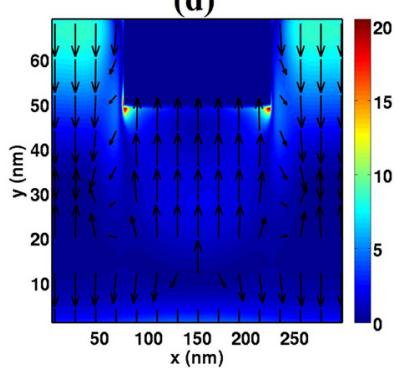

FIG. 5. The electrical results of the plasmonic OSC at the open-circuit condition. (a) Equipotential lines (V); (b) recombination rate with the logarithmic scale $\left(\mathrm{m}^{-3} \mathrm{~s}^{-1}\right)$; (c),(d) electron and hole current densities $\left(\mathrm{A} / \mathrm{m}^{2}\right)$. The color and arrow denote the amplitude and direction of the currents.

photocarrier generation is concentrated in this region and cannot be swept out efficiently with strong space charge effect. Remarkably, the different directions of the internal Efield make the plasmonic cell operate in the reverse and forward bias modes, respectively, for the center region below the grating ridge and the side regions below the grating troughs. As a result, the forward and reverse electron currents are obtained respectively at the side and center regions as drawn in Fig. 5(c). Likewise, Fig. 5(d) shows the forward and reverse hole currents respectively at the side and center regions. Distinguishing from this feature, the reverse electron current and the forward hole current have spatially uniform flow behaviors for the standard cell as shown in Figs. 4(c) and 4(d), respectively. Additionally, the internal E-field only points to one direction in the cross section of the standard cell.

Taking into account the electrical effects of the photogenerated carriers as described above, the short-circuit current of the plasmonic cell is improved by $13 \%$ due to the plasmon enhanced photoabsorption as depicted in Fig. 6. The characteristic parameters of OSCs are listed in the Table II. The slightly increased open-circuit voltage in the plasmonic OSC may be attributed to the favorable hole transport. A lot of holes are generated around the grating anode and can be collected efficiently. The FF is defined by the maximum power output over the product of short-circuit current and open-circuit voltage. A

TABLE I. The spatially averaged exciton dissociation probability ( $\langle$ Diss $\rangle$ ) and recombination loss $(\langle\operatorname{Rec}\rangle)$ at the short-circuit $(\mathrm{SC})$, maximum power (MP), and open-circuit (OC) conditions.

\begin{tabular}{lcccccc}
\hline \hline & $\begin{array}{c}\mathrm{SC} \\
\langle\mathrm{Diss}\rangle\end{array}$ & $\begin{array}{c}\mathrm{SC} \\
\langle\mathrm{Rec}\rangle\end{array}$ & $\begin{array}{c}\text { MP } \\
\langle\mathrm{Diss}\rangle\end{array}$ & $\begin{array}{c}\mathrm{MP} \\
\langle\mathrm{Rec}\rangle\end{array}$ & $\begin{array}{c}\mathrm{OC} \\
\langle\mathrm{Diss}\rangle\end{array}$ & $\begin{array}{c}\text { OC } \\
\langle\mathrm{Rec}\rangle\end{array}$ \\
\hline Standard (\%) & 69.83 & 0.88 & 62.34 & 9.19 & 59.69 & 82.56 \\
Plasmonic (\%) & 70.52 & 2.5 & 63.38 & 16.63 & 59.63 & 92.75 \\
\hline \hline
\end{tabular}




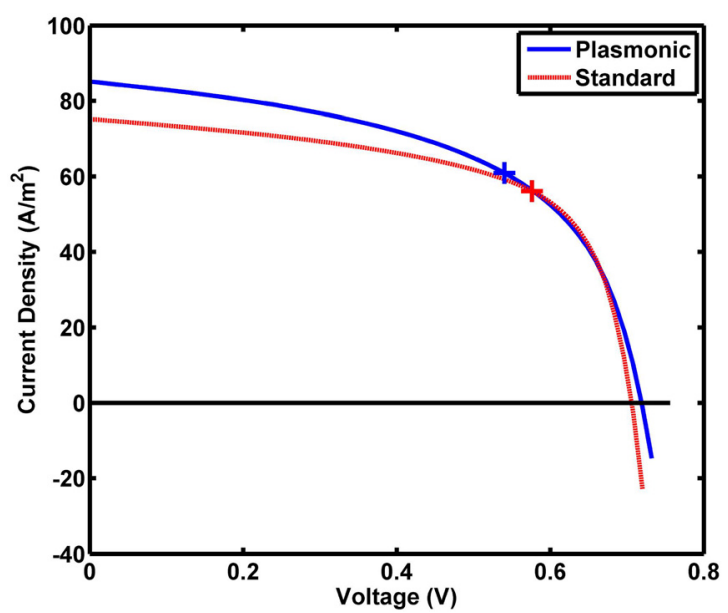

FIG. 6. The voltage-current density curve of the plasmonic and standard cells. The maximum power points are denoted by the plus signs.

significant $7 \%$ drop of the FF in the plasmonic cell is strongly confirmed by our multiphysics model. In particular, even if we change the periodicity $P$ and width $W$ of the grating as shown in Fig. 1, the dropped FF is observed universally. The dropped FF increases when the depth of the grating $H$ (in Fig. 1(b)) becomes shorter. Additionally, an optimum depth is required for achieving maximum enhancement in the optical absorption of the active material. The shunt resistance and ideal factor of the solar cell can be approximated by ${ }^{14}$

$$
\begin{gathered}
\left.\frac{d V}{d J}\right|_{J=J_{s c}} \approx R_{s h}, \\
\left.\frac{d V}{d J}\right|_{J=0, V=V_{o c}} \approx \frac{k_{B} T / q}{J_{s c}+J-V / R_{s h}} n+R_{s},
\end{gathered}
$$

where $J_{s c}$ is the short-circuit current, $V_{o c}$ is the open-circuit voltage, $n$ is the ideal factor, and $R_{s h}$ and $R_{s}$ are the shunt and series resistances, respectively. We fit the slopes of the above equations at the short-circuit current and open-circuit voltage points. We find that the shunt resistance is reduced and ideal factor is increased for the plasmonic cell. On one hand, the periodically modulated metallic grating excites the concentrated plasmonic waves near the anode resulting in nonuniform photocarrier generation. On the other hand, the modulated anode boundary is responsible for inhomogeneous built-in potential and internal E-field distributions below ridge and troughs of the grating anode, which has strong effects on the photocarrier transport and collections. In summary, the nonuniform photocarrier generation and transport are the physical origins of the dropped FF. The FF of the plasmonic cell may be improved by using sinusoidally modulated grating anode with smooth boundaries. Together with the improvement of the photogenerated current, the
TABLE II. The characteristic parameters of the standard and plasmonic OSCs involving short-circuit $\mathrm{J}_{\mathrm{sc}}$, open-circuit voltage $\mathrm{V}_{\mathrm{oc}}, \mathrm{MP}, \mathrm{FF}$, and PCE.

\begin{tabular}{lccccc}
\hline \hline & $\mathrm{J}_{\mathrm{sc}}\left(\mathrm{A} / \mathrm{m}^{2}\right)$ & $\mathrm{V}_{\mathrm{oc}}(\mathrm{V})$ & $\mathrm{MP}(\mathrm{W})$ & $\mathrm{FF}(\%)$ & PCE (\%) \\
\hline Standard & 75.18 & 0.706 & 32.34 & 60.91 & 3.23 \\
Plasmonic & 85.12 & 0.719 & 32.91 & 53.77 & 3.29 \\
\hline
\end{tabular}

power conversion efficiency could increase greatly. These cases will be addressed in the future.

We have investigated the plasmonic OSC with the metallic rectangular-grating anode through the multiphysics solutions to Maxwell's equations and semiconductor equations. The grating anode induces nonuniform optical absorption and inhomogeneous internal E-field distribution. Thus uneven photocarrier generation and transport are formed in the plasmonic OSC leading to the dropped FF. The multiphysics modeling and understanding are fundamentally important for improving the performance of organic photovoltaics.

The authors acknowledge the support of the grants (Nos. 712010, 711609, and 711511) from the Research Grant Council of the Hong Kong, the grant (No. 10401466) from the University Grant Council (UGC) of the University of Hong Kong, and the Small Project Funding of the University of Hong Kong (No. 201109176133). This project is also supported by the UGC of Hong Kong (No. AoE/P-04/08), by The National Natural Science Foundation of China (No. 61201122), and in part by a Hong Kong UGC Special Equipment Grant (SEG HKU09).

${ }^{1}$ C. J. Brabec, S. Gowrisanker, J. J. M. Halls, D. Laird, S. J. Jia, and S. P. Williams, Adv. Mater. 22, 3839-3856 (2010).

${ }^{2}$ C. Deibel and V. Dyakonov, Rep. Prog. Phys. 73, 096401 (2010).

${ }^{3}$ H. A. Atwater and A. Polman, Nat. Mater. 9, 205 (2010).

${ }^{4}$ R. A. Pala, J. White, E. Barnard, J. Liu, and M. L. Brongersma, Adv. Mater. 21, 3504-3509 (2009).

${ }^{5}$ C. J. Min, J. Li, G. Veronis, J. Y. Lee, S. H. Fan, and P. Peumans, Appl. Phys. Lett. 96, 133302 (2010).

${ }^{6}$ W. E. I. Sha, W. C. H. Choy, Y. G. Liu, and W. C. Chew, Appl. Phys. Lett. 99, 113304 (2011).

${ }^{7}$ L. J. A. Koster, E. C. P. Smits, V. D. Mihailetchi, and P. W. M. Blom, Phys. Rev. B 72, 085205 (2005).

${ }^{8}$ V. D. Mihailetchi, J. Wildeman, and P. W. M. Blom, Phys. Rev. Lett. 94, 126602 (2005).

9J.-Y. Wang, F.-J. Tsai, J.-J. Huang, C.-Y. Chen, N. Li, Y.-W. Kiang, and C. C. Yang, Opt. Express 18, 2682-2694 (2010).

${ }^{10}$ X. Li, N. P. Hylton, V. Giannini, K.-H. Lee, N. J. Ekins-Daukes, and S. A. Maier, Opt. Express 19, A888-A896 (2011).

${ }^{11}$ M. G. Deceglie, V. E. Ferry, A. P. Alivisatos, and H. A. Atwater, Nano Lett. 12, 2894-2900 (2012).

${ }^{12}$ W. E. I. Sha, W. C. H. Choy, Y. Wu, and W. C. Chew, Opt. Express 20, 2572-2580 (2012).

${ }^{13}$ See supplementary material at http://dx.doi.org/10.1063/1.4768236 for the algorithm details, modeling parameters, and simulation results.

${ }^{14}$ C. Zhang, J. Zhang, Y. Hao, Z. Lin, and C. Zhu, J. Appl. Phys. 110, 064504 (2011). 